\title{
Das Versprechen von der Steigerung der Leistungsfähigkeit
}

\author{
Mit Converging Technologies ist das Ver- \\ sprechen verbunden, dass Menschen durch \\ ihre Anwendungen leistungsfähiger werden. \\ In welche Richtung geht eine Innovationspolitik, \\ die auf die Wettbewerbsfähigkeit der Einzelnen \\ setzt? \\ Von Petra Schaper-Rinkel
}

\begin{abstract}
Im Jahr 2001 veranstaltete die US-National Science Foundation die erste einer Reihe innovationspolitischer Konferenzen, deren Begriffe sich schnell in kontroversen Debatten wiederfanden. Unter dem Titel „Converging Technologies for Improving Human Performance“ wurde die Konvergenz von Nanotechnologie, Biotechnologie, Informationstechnologien und Kognitionswissenschaften als technische Wunscherfüllungsmaschine des 21. Jahrhunderts annonciert (Roco/Bainbridge 2002). Durch diese Konvergenz könnte die individuelle und gesellschaftliche Leistungsfähigkeit enorm gesteigert werden. Ziel der Optimierung ist dabei ganz traditionell die internationale Wettbewerbsfähigkeit der amerikanischen Wirtschaft.
\end{abstract}

\section{Diskurse um die Technik des 21. Jahrhunderts}

2003 etablierte die Europäische Kommission eine Expertengruppe zur Erarbeitung eines europäischen Ansatzes. Während die US-Strategie Individuen durch technische Innovationen $\mathrm{zu}$ optimieren sucht, setzt die Europäische Strategie darauf, das technologische Potential konvergierender Technologien durch soziale Innovationen zu realisieren (Nordmann 2007). Trotz einer vorsichtigen Relativierung bleibt auch die europäische Wettbewerbsfähigkeit der Referenzpunkt (Hochrangige Expertengruppe 2004).

Neu sind einige der Ansatzpunkte für die Innovationspolitik des 21. Jahrhundert. Zum einen richtet sie sich konzeptionell direkt auch auf die Individuen selbst. Prägnant zusammengefasst wurde dieser Anspruch in einem Beitrag auf einer der Folgekonferenzen, auf denen es hieß, die technologisch optimierte mentale Gesundheit würde zur entscheidenden Waffe im Wettbewerb werden, da entsprechend optimierte Individuen dank ihres neuro-kompetitiven Vorteils produktiver sein würden (Lynch 2004). Zum anderen lässt sich eine immer frühere Integration von heterogenen Akteuren in die innovationspoliti- sche Szenarien-Entwicklung feststellen, eine Art Innovationspolitik zum Mitmachen hinsichtlich der Visionen.

Was es zukünftig mit der Verschmelzung von Technologien und Forschungsfeldern auf sich haben könnte, bleibt bisher wissenschaftlich und technisch unklar. Doch gerade die Vagheit des Konzepts behindert die involvierten Akteure nicht; sie bietet vielmehr eine Einladung zu einer Innovations-Mitmachpolitik, die im Folgenden skizziert wird. Im Zentrum der Darstellung können daher nur Verschiebungen innovationspolitischer Diskurse stehen, wobei Diskurse in diesem Fall nicht die Argumentationen sind, sondern vielmehr die politischen umfassenden Prozesse, in denen die vielbeschworene Frage der Technik im 21. Jahrhundert im nach wie vor ungelösten Widerspruch von beschleunigtem wirtschaftlichen Wachstum und Nachhaltigkeitsansprüchen steht.

Dieser Widerspruch ist im Konzept der CT nicht mehr vorhanden, denn sie würden nicht nur die menschlichen Kapazitäten im militärischen Bereich erhöhen, sondern zugleich zur Erreichung von Nachhaltigkeitszielen beitragen und außerdem den physischen und kognitiven Niedergang aufhalten, der mit der alternden Gesellschaft verknüpft ist (Roco/Bainbridge 2002). Mit dem technikdeterministischen Automatismus, in dem Technologien eine unspezifisch positive Wirkung zugeschrieben wird, verschwindet, dass Technologien durch spezifische Anforderungen entwickelt werden. Die wachstumsorientierte Ausrichtung in Form einer technologischen Aufrüstung von Individuen forciert eine Technologieentwicklung, die auf verschärfte Konkurrenz setzt und nicht auf Kooperation. Sie stellt zudem eine Intensivierung von Ausbeutung dar, indem Individuen auf der Ebene der direkten Optimierung ihrer körperlichen und mentalen Kapazitäten angerufen werden, sich für einen verschärften Wettbewerb aufzurüsten. So verdeckt die Rede davon, dass Technologien Probleme lösen werden, genau die politische Kontroverse, wie Technik konstituiert und regiert werden müsste, um nicht nur rhetorisch, sondern materiell ökologischen und sozialen Nachhaltigkeitszielen zu entsprechen.

\section{Innovationspolitische Anrufung der Individuen}

Neben militärischen Szenarien optimierter Soldaten, die durch die Verbindung von Informations- und Telekommunikationstechnik mit avancierten Neurotechnologien die entscheidende Millisekunde früher gedankengesteuert feuern können, wurden auf der ersten Konferenz zu Converging Technologies auch Überlegungen kritischer Forscherinnen wie Sherry Turkle 
integriert, die analysiert, wie sich die Grenzen zwischen Menschen und Maschinen auflösen, wenn Roboter Spielgefährten für Kinder werden oder wenn Maschinen von Werkzeugen zu interaktiven Gefährt(inn)en werden (Roco/Bainbridge 2002). Die Spannbreite derjenigen, die zur Technologieentwicklung des 21. Jahrhunderts beitragen sollen, mag auf den ersten Blick verwunderlich erscheinen, lässt sich aber auch als ein Indiz dafür lesen, dass Partizipation heute zu einem integralen Modus staatlicher Innovationspolitik geworden ist. Eine frühe Integration von sozialwissenschaftlicher Forschung verweist darauf, dass die antizipierte Nutzung von Technologien auf der Ebene der Visionen Teil der Governance von Zukunftstechnologien wird.

Richtete sich die Technologie- und Innovationspolitik der Vergangenheit auf die institutionellen Strukturen von Wissenschaft und Wirtschaft und suchte diese durch Technologiezentren, Kompetenzzentren, Vernetzungsinitiativen und die unternehmerische Bildung junger Wissenschaftler(innen) zu optimieren, so ist das Neue der konvergierenden Technologien, dass sie direkte technologische Leistungssteigerung von Individuen versprechen und fordern. Innovationspolitik weitet sich mit Konvergenz-Konzepten aus. Adressat der Technologiepolitik sind nicht mehr nur Wissenschaft, Industrie und Wirtschaft, sondern auch die Individuen, die als zukünftige Produzent(inn)en und Nutzer(innen) auf das Kommende vorbereitet werden. Zugleich wird die Logik des Wettkampfes als Treiber von technologischer Entwicklung ausgeweitet. Nicht mehr nur Unternehmen sind gehalten, sich mit Nano-Bio-Info-Kogno auf dem Stand der Dinge zu halten und zukünftige Entwicklungen zu antizipieren. Der Zwang zur Selbstoptimierung wird nun auf der technologischen Ebene zum Treiber für Individuen mit den technischen Artefakten zu konvergieren. Und zwar passiert dies nicht selbstgewählt. Denn die gesellschaftliche Arbeitsteilung und die entsprechenden gesellschaftlichen Anforderungen produzieren einen spezifischen Optimierungsdruck, so dass sich jede und jeder als technologisch optimierungsbedürftig erweist.

\section{Innovationspolitik zum Mitmachen}

Technologiepolitik blieb lange einem linearen Konzept technischer Entwicklung verhaftet, wie sie seit Francis Bacon gesehen wird. Der Grundlagenforschung folgt die angewandte Forschung, die wiederum in Produkt- und Verfahrensinnovationen mündet. Politik hat in diesem Konzept die Aufgabe, der Eigengesetzlichkeit der Grundlagenforschung Mittel zur Verfügung zu stellen, und erst in den Stadien danach wird Forschung auf Märkte oder auch gesellschaftliche Bedürfnisse ausgerichtet. Heute ergeht auch an Grundlagenforschung der Auftrag, sich auf Felder auszurichten, die einen hohen ökonomischen und manchmal auch gesellschaftlichen Nutzen aufweisen sollen.

Converging Technologies lassen sich als ein technologiepolitisches Experiment lesen, das bereits im Vorfeld jeglicher Forschungsförderung zum Mitmachen einlädt - wobei Mitmachen nicht Mitbestimmen meint. In der klassischen Technologiepo- litik standen Technologien im Zentrum, die linear gedacht in Anwendungen und Märkte münden sollten. In der darauf folgenden Innovationspolitik seit Ende der 1980er Jahre wurden Netzwerke, Technologiezentren, Kompetenzzentren und Technologieparks gefördert, um unterschiedliche Akteure aus Grundlagenforschung, angewandter Forschung, Wirtschaft und Investoren zusammenzubringen, damit sie in interaktiven und kreativen Auseinandersetzungen innovieren mögen.

\section{Förderung etablierter Ansätze}

Konzeptionell wurde damit der Akteurskreis auch von Förderung ausgeweitet, nicht mehr nur etablierte Industrieunternehmen standen im Fokus der Politik, sondern auch innovative Einzelunternehmer(innen). Wissenschaftler(innen) sahen sich in vielfältigen Programmen aufgefordert, Unternehmer(innen) zu werden, ihre Forschung in Patente umzusetzen und daraus wirtschaftlich erfolgreiche Unternehmen zu machen. Parallel zur technologiepolitisch finanzierten Vernetzung aller Akteure zur Kommerzialisierung von wissenschaftlichem Wissen wurden in den neunziger Jahren auch Visionen zu einem Modus von Innovationspolitik. Die Nanotechnologiepolitik ist das zentrale Beispiel für eine Visions-getriebene Innovationspolitik, die in eine Ökonomie des Versprechens mündet, in der widersprüchliche gesellschaftliche Interessen dank einer Zukunftstechnologie zum Wohle aller versöhnt werden sollen ohne dass eine entsprechende Technologiepolitik in diese Richtung ging (Schaper-Rinkel 2006). Auf die öffentlichen Forschungsausgaben bezogen, waren es weder revolutionäre Forschungsansätze noch Umwelttechnologien, die primär von den Förderprogrammen profitierten, sondern vielmehr die etablierten Industrien. In der Automobilbranche wie der Mikroelektronik sollte dank Nanotechnologie die Wettbewerbsfähigkeit gesteigert werden und dank Effizienzsteigerung auch noch etwas für die Umwelt getan werden. Während die erwarteten wirtschaftlichen Effekte in Form von Marktszenarien Teil der Förderanträge auf öffentliche Gelder darstellen, sind Szenarien der erwarteten positiven Umweltwirkungen kein Gegenstand der Bewertung von Förderanträgen.

Bei allen Visionen blieb es dabei, dass Innovationspolitik von einer Technologie ausging. Bei dem Konzept der CT ist es dagegen die Vernetzung von Forschungsfeldern, die zukünftig die wahren Innovationen bringen möge. Die Offenheit des CT-Ansatzes entspricht den neuen Innovationskonzepten und -theorien, die ebenfalls das Mitmachen und die Integration von Unterschiedlichem favorisieren: Im Open Innovation-Modell werden Innovationen nicht mehr aus den abgeschotteten Forschungsabteilungen von Großunternehmen erwartet, sondern von der Interaktion zwischen Unternehmen und unternehmensexternen Akteuren (Chesbrough 2003). Nutzer(innen) und Produzent(inn)en von Innovationen sind nicht mehr als getrennte Gruppen gedacht, vielmehr geht der Ansatz der Lead user Innovation davon aus, dass Konsument(inn)en Produkte aktiv gestalten und erfinden und Unternehmen davon syste- $\rightarrow$ 


\section{„Bei steigender Partizipation, also steigender Anzahl von beteiligten Akteuren, sinkt der Einfluss jedes Einzelnen."}

matisch lernen können (von Hippel 2006). Die breite und frühe Beteiligung von Akteuren aus Wissenschaft, Wirtschaft und Politik ist analog dazu eine Open Innovation-Policy, sie konstituiert erst einen möglichen Adressatenkreis für potentielle Forschungsprogramme der Zukunft und aktiviert eine Vielzahl von Akteuren, Teil innovationspolitischer Aktivitäten zu werden, deren konkrete Ausgestaltung unbestimmt ist.

\section{Die politische Zukunft von Converging Technologies}

Im Gegensatz zur Nanotechnologie, wo innovationspolitischer Diskurs und öffentliche Programme zur Forschungsförderung zeitgleich ein wachsendes Interesse in Gang setzten, blieb es bei Converging Technologies bisher weitgehend bei Konzepten, denen keine großvolumigen Förderprogramme folgten. Was im Umfeld der Converging Technologies konzeptionell begonnen wurde, findet sich heute allerdings in bereits transformierter Form in anderen Forschungs-Agenden. Das Karussell der jeweils als neu apostrophierten Zukunftstechnologien, so ließe sich es sich polemisch zuspitzen, hat sich so schnell gedreht, dass das Konzept einer allumfassenden Konvergenz unbemerkt ausgestiegen ist. Gleichermaßen ersetzt, modifiziert und konkretisiert wird es heute insbesondere in der Synthetischen Biologie wie auch im Forschungsfeld der Human Enhancement Technologies. Synthetische Biologie war kein explizit genanntes Forschungsfeld des Konvergenz-Diskurses, bedeutet jedoch eine weitere Steigerung des CT-Konzepts im Sinne eines ingenieur- und technikwissenschaftlichen Zugangs zu lebender Materie. Ziel der Synthetischen Biologie ist es nicht nur, genetische Information für spezifische Stoffwechselvorgänge in standardisierter Form als Bausteine zu konfigurieren und sie in dieser Weise für technische Anwendungen zu nutzen, sondern zudem Mikro-Organismen zu programmieren und damit neues Leben zu erfinden.

\section{Das Partizipations-Paradox}

Die Forderung nach partizipativer Gestaltung von Technologien und Technologiefeldern unter Beteiligung von unterschiedlichen Akteuren in einem frühen Stadium ist nicht zuletzt aus der Tradition der interdisziplinären Technikforschung entstanden, die Technik als sozial konstituiert und damit gestaltbar ansieht (Bijker et al. 1987; MacKenzie/Wajcman 1999). Diese Forderung kam zudem aus der Umweltforschung, die postulierte, dass sich mit partizipativen Mechanismen qualitativ bessere Ergebnisse in Entscheidungsprozessen erzielen lassen, indem in den Prozess unterschiedliche Perspektiven einbezogen sind (Fiorino 1990). Wenn Partizipation heute als paradox kritisiert wird, so zielt die Kritik darauf ab, dass bei steigender Anzahl der beteiligten Akteure der Einfluss der einzelnen Beteiligten sinkt. Für die so genannten Converging Technologies könnte sich das Paradox folgendermaßen spezifizieren. Bei steigender Beteiligung heterogener Akteure steigt die rhetorische Integration von Ansprüchen insbesondere in Bezug auf Nachhaltigkeit in der Innovationspolitik bei gleichzeitig sinkender Konkretisierung eben dieser Ansprüche. Sie zu konkretisieren ist eine Frage von politischen Machtverhältnissen - nicht von technischen Wissensbeständen.

\section{Literatur}

Bijker, W. E. / Hughes, T. P. / Pinch, T. (eds.): The Social Construction of Technological Systems. New Directions in the Sociology and History of Technology. Cambridge 1987.

Chesbrough, H. W.: The Era of Open Innovation. In: MIT Sloan Management Review 44, 3/2003. S. 35-41

Fiorino, D. J.: Citizen Partizipation and Environmental Risk: A Survey of Institutional Mechanismen. In: Science, Technology, \& Human Values 15, 2/1990. S. 226-43.

Hochrangige Expertengruppe, HLEG: Technologische Konvergenz und die Zukunft der europäischen Gesellschaften. Foresight zur neuen Technologiewelle. In: Nordmann, A. (ed.): Berichterstattung. Brüssel 2004.

Lynch, Z.: Neurotechnology and Society (2010-2060). In: Roco, M. C. Montemagno, C. D. (eds.): Co-evolution of Human Potential and Converging Technologies. New York 2004. S. 229-33.

MacKenzie, D. / Wajcman, J. (eds.): The Social Shaping of Technology. Buckingham 1999.

Nordmann, A: Knots and Strands: An Argument for Productive Disillusionment. In: Journal of Medicine \& Philosophy 32, 3/2007, S. 217-36.

Roco, M. C. / Bainbridge, W. S.: Converging Technologies for Improving Human Performance. Nanotechnology, Biotechnology, Information Technology and Cognitive Science. Arlington 2002.

Schaper-Rinkel, P.: Governance von Zukunftsversprechen: Zur politischen Ökonomie der Nanotechnologie. In: PROKLA 145 „Ökonomie der Technik“ 36, 4/2006. S. 473-96.

Von Hippel, E.: Democratizing Innovation. Cambridge 2006.

\section{AUTORIN + KONTAKT}

Dr. Petra Schaper-Rinkel ist Senior Researcher am Department Foresight \& Policy Development am Austrian Institute of Technology AIT.

Austrian Institute of Technology, Foresight \& Policy Development Department, Donau-City-Straße 1,

1220 Wien, Österreich. Tel.: +4350550-4562, E-Mail: Petra.Schaper-Rinkel@ait.ac.at 
(c) 20I0 Authors; licensee IÖW and oekom verlag. This is an article distributed under the terms of the Creative Commons Attribution Non-Commercial No Derivates License (http://creativecommons.org/licenses/by-nc-nd/3.o/), which permits unrestricted use, distribution, and reproduction in any medium, provided the original work is properly cited. 